University of Nebraska - Lincoln

DigitalCommons@University of Nebraska - Lincoln

September 1984

\title{
Magnetic properties and chemical short-range order in Fe-Pd- based metallic glasses
}

\author{
J.G. Zhao \\ University of Nebraska - Lincoln \\ David J. Sellmyer \\ University of Nebraska-Lincoln, dsellmyer@unl.edu
}

Follow this and additional works at: https://digitalcommons.unl.edu/physicssellmyer

Part of the Physics Commons

Zhao, J.G. and Sellmyer, David J., "Magnetic properties and chemical short-range order in Fe-Pd-based metallic glasses " (1984). David Sellmyer Publications. 150.

https://digitalcommons.unl.edu/physicssellmyer/150

This Article is brought to you for free and open access by the Research Papers in Physics and Astronomy at DigitalCommons@University of Nebraska - Lincoln. It has been accepted for inclusion in David Sellmyer Publications by an authorized administrator of DigitalCommons@University of Nebraska - Lincoln. 


\title{
Magnetic properties and chemical short-range order in Fe-Pd-based metallic glasses
}

\author{
J. G. Zhao* and D. J. Sellmyer \\ Behlen Laboratory of Physics, University of Nebraska, Lincoln, Nebraska 68588
}

(Received 11 October 1983)

\begin{abstract}
High-field magnetization and ac susceptibility measurements are reported on a series of $\mathrm{Fe}_{x} \mathrm{Pd}_{80-x} \mathrm{Si}_{10} \mathrm{~B}_{10}$ rapidly quenched alloys for $0 \leqslant x \leqslant 80$. X-ray diffraction data show that these alloys are amorphous up to at least $x=40$ and that the $x=60$ and 80 samples are partially crystalline. The ac susceptibility data can be interpreted in terms of ferromagnetic, spin-glass, and double transitions, and a qualitative magnetic phase diagram of the system is obtained. The concentration dependence of the saturation magnetization, as well as the magnetic phase diagram, exhibits sharp anomalies at about $x=13$. These data suggest the presence of chemical short-range order for $x \geq 13$. X-ray photoelectron and Mössbauer results are consistent with this model.
\end{abstract}

\section{INTRODUCTION}

Magnetic properties of amorphous $\mathrm{Fe}_{x} \mathrm{Pd}_{80-x} \mathrm{Si}_{20}$ alloys and similar compositions have been reported by a number of authors. In most cases the $\mathrm{Fe}$ content was less than 25 at. \%. Early magnetization data on $a-\mathrm{Fe}_{x} \mathrm{Pd}_{80-x} \mathrm{Si}_{20}(x \leqslant 7)$ were interpreted by a mechanism of spin polarization of the $\mathrm{Pd}$ atoms by neighboring $\mathrm{Fe}$ atoms. ${ }^{1}$ Then Nagel, Fisher, Tauc, and Bagley ${ }^{2}$ suggested that in $a$-PdCuSi alloys electron transfer from Si to Pd effectively fills the $d$ holes of Pd so that in $a$-FePdSi alloys the polarization of $\mathrm{Pd}$ atoms would be unlikely. But the relatively large electronic heat capacity $^{3}$ and more recent photoemission measurements ${ }^{4}$ on $\mathrm{Pd}-\mathrm{Si}$ glasses indicated the existence of appreciable $\mathrm{Pd} d$ band states below the Fermi level $\left(E_{F}\right)$ so that some amount of polarization of the $\mathrm{Pd}$ atoms by $\mathrm{Fe}$ moments is expected.

For small $\mathrm{Fe}$ concentrations Mössbauer ${ }^{5}$ and ac susceptibility data ${ }^{6}$ showed the existence of transitions with spinglass character. Dublon and co-workers studied magnetic properties of the $a-\mathrm{Fe}_{x} \mathrm{Pd}_{82-x} \mathrm{Si}_{18}$ system for $x \leq 25$ and determined the magnetic phase diagram and critical exponents for this system..$^{7-9}$

We report here a study of the magnetic properties of rapidly quenched $\mathrm{Fe}_{x} \mathrm{Pd}_{80-x} \mathrm{Si}_{10} \mathrm{~B}_{10}$ alloys with $0 \leqslant x \leqslant 80$. We have found in many other systems that the addition of about 10 at. \% boron facilitates the production of an amorphous phase. This was successful in part in this series since the range of completely amorphous samples was extended to Fe concentrations at least up to 40 at. \%. Our interest has been in the study of a system in which exchange fluctuations become increasingly important as the moment concentration decreases so that the possibility exists of double transitions (paramagnetic-ferromagnetic and ferromagneticspin-glass). Also, we wished to investigate $\mathrm{Pd}$ spin polarization and chemical short-range order in transition-metalmetalloid glasses.

\section{EXPERIMENTAL METHODS}

Amorphous $\mathrm{Fe}_{x} \mathrm{Pd}_{80-x} \mathrm{Si}_{10} \mathrm{~B}_{10}$ alloys $(0 \leqslant x \leqslant 80)$ were prepared by splat cooling arc-melted droplets. The foil-like samples that were obtained, typically about $0.005 \mathrm{~cm}$ thick and $2.5 \mathrm{~cm}$ in diameter, were studied with high-resolution
Mo $K \alpha$ x-ray diffraction measurements; a $\mathrm{Si}(\mathrm{Li})$ solid-state detector was employed. For $x \leqslant 40$ the diffraction patterns were liquidlike and thus the samples appeared to be completely amorphous. However, for $x=60$ and $x=80$ some crystalline lines were present so that it appears that the cooling rate achieved in our splat-cooling facility is not sufficiently high to produce homogeneous glasses for these high-Fe concentrations. Nevertheless, some magnetization data will be reported for these two concentrations in the following, with the structure noted in the figure.

ac susceptibility measurements were performed with a balanced pair of coils and with a driving field of about $1 \mathrm{Oe}$ at a frequency of $280 \mathrm{~Hz}$. Standard phase-sensitive detection techniques, a probe slowly dipped into a helium bath, and a microprocessor-controlled data acquisition system were employed. Magnetization measurements at $4.2 \mathrm{~K}$ were made in fields up to $80 \mathrm{kOe}$ with a homemade vibrating sample magnetometer. This system has been described elsewhere. ${ }^{10}$

$\mathrm{X}$-ray photoemission data were taken with an ESCA LAB-5 model spectrometer at the Academia Sinica Institute of Physics in Beijing. Before taking the valence-band spectra, with $\mathrm{Al} K \alpha$ radiation, the high-energy core-level spectra were checked to make sure that no carbon and oxygen lines were present.

\section{RESULTS AND DISCUSSION}

\section{A. Magnetic phase diagram}

Figure 1 shows the results of the ac susceptibility reasurements for all of the amorphous samples. Several interesting features are evident. Some relatively easily understood aspects are the sharp transition for $x=5$, suggesting a spin-glass transition, and the essentially flat response for $x=40$ over the whole temperature range. This latter curve indicates a ferromagnetic sample with $T_{c}>294 \mathrm{~K}$, and a demagnetization-limited ac susceptibility. For $x=10$ there is a sharp peak at about $60 \mathrm{~K}$ suggesting a paraferromagnetic transition, and a relatively sharp downturn at about $37.5 \mathrm{~K}$. Given the apparent transition to a spin-glass phase for $x=5$ and the Sherrington-Kirkpatrick the ory ${ }^{11}$ for systems with competing ferromagnetic and spin-glass order, it is reasonable to assume that the transition at $37.5 \mathrm{~K}$ is a ferromagnetic-spin-glass transition, sometimes called a 


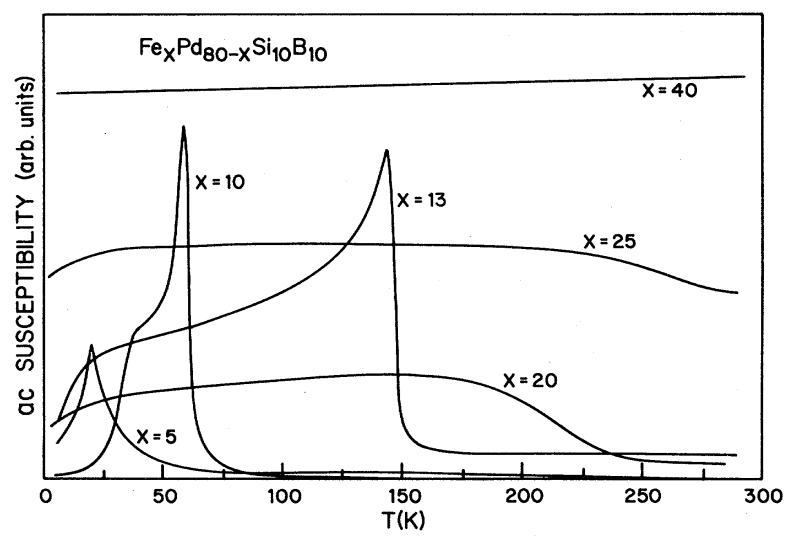

FIG. 1. ac susceptibility vs temperature for several Fe concentrations.

"reentrant" transition at $T_{f g}$. The sharply decreasing ac susceptibility for $x=10$ just below $T_{c}$ is not easy to interpret, but it may be related to domain walls becoming increasingly sluggish as a result of defects, as the temperature is lowered. The $x=13$ sample has behavior qualitatively similar to that of $x=10$, but $T_{c}$ and $T_{f g}$ are farther apart. For $x=20$ and 25 the two transitions, if they exist, become increasingly hard to define because of the lack of sharp structure in the data. However, such data are not uncommon for systems with double transitions. ${ }^{12}$ If we simply define $T_{c}$ to be at the midpoint of the rise in $\chi_{\mathrm{ac}}$ and $T_{f g}$ to be at the intersection of straight-line extrapolations of the data above and below $T_{f g}$, we obtain the qualitative phase diagram shown in Fig. 2. As noted above for the $x=20$ and 25 samples the determination of $T_{c}$ is subject to considerable error; nevertheless, when coupled with the more accu-

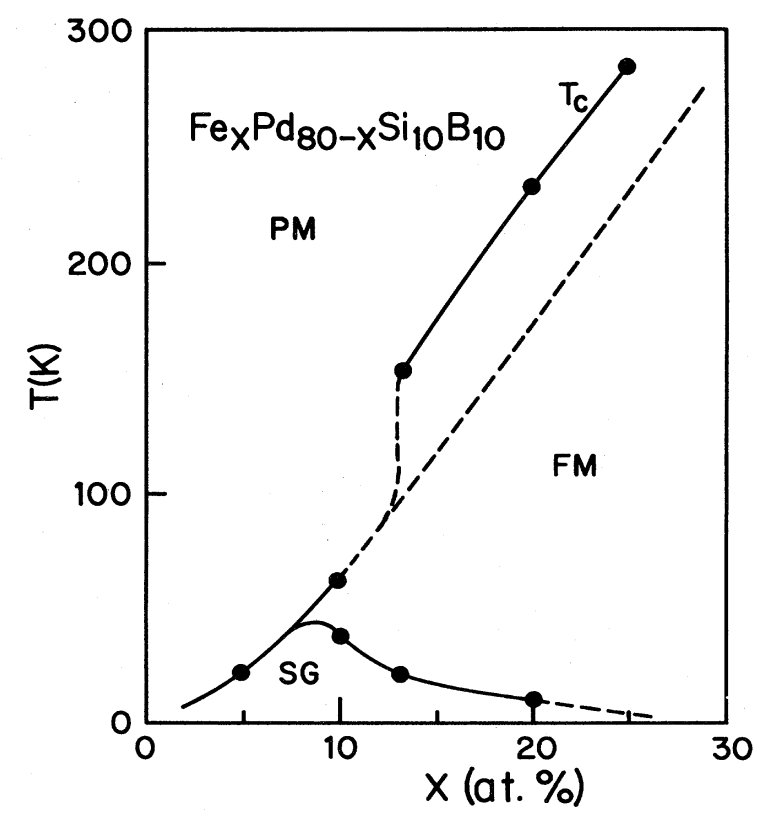

FIG. 2. Qualitative magnetic phase diagram showing paramagnetic (PM), ferromagnetic (FM), and spin-glass (SG) regions. The dashed line is simply an extrapolation of the $T_{c}(x)$ curve for small $x$ values (see text). rate $T_{c}$ measurement for $x=13$, it appears as if there is an upward shift from an extrapolation of the data for lower concentrations. A similar kink was seen in the initial $T_{c}(x)$ data for the related $\mathrm{Fe}_{x} \mathrm{Pd}_{82-x} \mathrm{Si}_{18}$ system. ${ }^{8}$ But later work on the same system, in which the analysis was performed in a different way, showed a much smaller kink near $x=15 .^{9}$ Our data are suggestive, at least, of a change in the chemical short-range order (CSRO) or clustering near $x=13$. Further evidence for such CSRO will be discussed in Sec. II B.

\section{B. Polarization of Pd atoms}

Magnetization data to $80 \mathrm{kOe}$ and at $4.2 \mathrm{~K}$ are shown for the wholly amorphous samples $(x \leqslant 40)$ in Fig. 3. Data for the partially crystalline samples, $x=60$ and 80 , are not included in this figure but will be used in subsequent plots because there are no apparent problems in understanding how the $x=60$ and $x=80$ data match onto the lower Fe concentration data.

Figure 4 shows the composition dependence of the magnetization. Curve $\mathrm{A}$ is the saturation moment per formula unit, here defined to be the moment at $80 \mathrm{kOe}$. This curve also shows structure at about $x=13$. Similar structure was found near $x=10$ in the $\mathrm{Fe}_{x} \mathrm{Pd}_{82-x} \mathrm{Si}_{18}$ system. ${ }^{7}$ In the case of $\mathrm{Fe}_{80} \mathrm{Si}_{10} \mathrm{~B}_{10}$ the moments clearly are associated only with $\mathrm{Fe}$ atoms, and the moment per $\mathrm{Fe}$ atom is $\mu_{\mathrm{Fe}}=2.0 \mu_{B}$. This value agrees well with that obtained from Mössbauer hyperfine spectra of $\mathrm{Fe}_{x} \mathrm{Pd}_{80-x} \mathrm{Si}_{20}$ (Ref. 13) and other results. If we then assume that each $\mathrm{Fe}$ atom has a moment of $2.0 \mu_{B}$ for all $x$, curve B of Fig. 4 is obtained. The difference between curves $A$ and $B$ is plotted as curve $C$ and this is reasonably attributed to the Pd moment per formula unit, $\mu(\mathrm{Pd})$, induced by neighboring $\mathrm{Fe}$ atoms. Clearly, $\mu(\mathrm{Pd})$ will be zero at $x=0$ and $x=80$ since no Pd atoms can be polarized. It is also possible to understand why $\mu(\mathrm{Pd})$ has a maximum value at about $x=40$. If it is assumed that a Pd atom must have at least one $\mathrm{Fe}$ nearest neighbor to develop a spin polarization, then one can calculate, using random occupancy of sites, the average number of $\mathrm{Pd}$ atoms with a spin polarization. Such a calculation leads to a maximum value of the average moment per Pd atom of about $0.4 \mu_{B}$, at a concentration of about $x=40$. This model is not definitive because of the assumptions entering it, but at least is capable of rationalizing the general increase and then decrease in the moment attributable to spin-polarized Pd

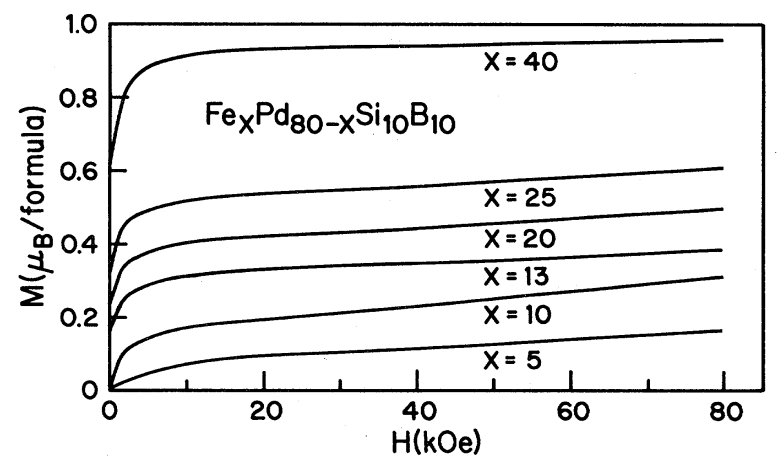

FIG. 3. Magnetization vs applied field at $4.2 \mathrm{~K}$ for different $\mathrm{Fe}$ concentrations. 


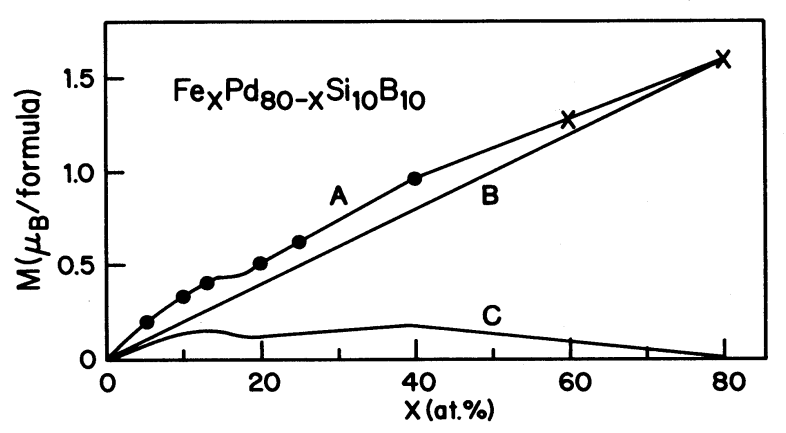

FIG. 4. Magnetic moment per formula unit: Curve A: Total moment. $x=60$ and 80 samples are partially crystalline. Curve B: Moment assumed to be associated with $\mathrm{Fe}$ atoms, each with $2.0 \mu_{B}$. Curve C: Difference of curves A and B, moment associated with Pd atoms due to spin polarization by neighboring $\mathrm{Fe}$ atoms.

atoms. Of course, it implies nothing about the structure in $\mu(\mathrm{Pd})$ seen near $x=13$. This structure, when coupled with the anomalies seen at the same composition in the magnetic phase diagram (Fig. 2), gives stronger evidence that CSRO or perhaps even a phase separation into two glassy phases is occurring in this system.

\section{Photoemission and Mőssbauer measurements}

The valence-band $\mathrm{x}$-ray photoemission spectroscopy spectra for $x=5,10,15,20$, and 25 were measured and a typical spectrum, for $x=15$, is shown in Fig. 5(a). The occupied levels near $E_{F}$ are mainly $\mathrm{Fe} 3 d$ and $\mathrm{Pd} 4 d$ states. The crosshatched area in Fig. 5(a) is the electron distribution curve above $E_{F}$ due to the spectrometer resolution $(\sim 1$ $\mathrm{eV})$. The ratio of the crosshatched area to the total area under the valence-band spectrum should give a qualitative representation, as a function of $x$, of the concentration dependence of the $d$ electron count near $E_{F}$. This ratio is plotted in Fig. 5(b), where a minimum is seen at $x=15$. It is not possible to infer anything about CSRO from these measurements, but the change in slope at $x=15$ seen in Fig. 5(b) again is consistent with a change in CSRO as discussed above in connection with structure seen in the magnetic phase diagram and magnetization at about this composition.

Some limited Mössbauer results ${ }^{14}$ also support the idea of significant CSRO or phase separation above $x \simeq 13$. The spectrum for $\mathrm{Fe}_{20} \mathrm{Pd}_{60} \mathrm{Si}_{10} \mathrm{~B}_{10}$ at both 77 and $4.2 \mathrm{~K}$ shows a magnetic hyperfine split spectrum plus a doublet attributable to a quadrupole interaction. The persistence of the quadrupole spectrum below the magnetic ordering temperature indicates that some of the $\mathrm{Fe}$ atoms do not experience a magnetic hyperfine field, and are thus in a paramagnetic or very weakly magnetic region.

In summary, our results on the amorphous $\mathrm{Fe}_{x} \mathrm{Pd}_{80-x} \mathrm{Si}_{10} \mathrm{~B}_{10}$ system show that glassy alloys can be obtained up to about $x=40$. A magnetic phase diagram has been obtained including paramagnetic, ferromagnetic, and spin-glass regions, and for a limited region of $x$ double tran-
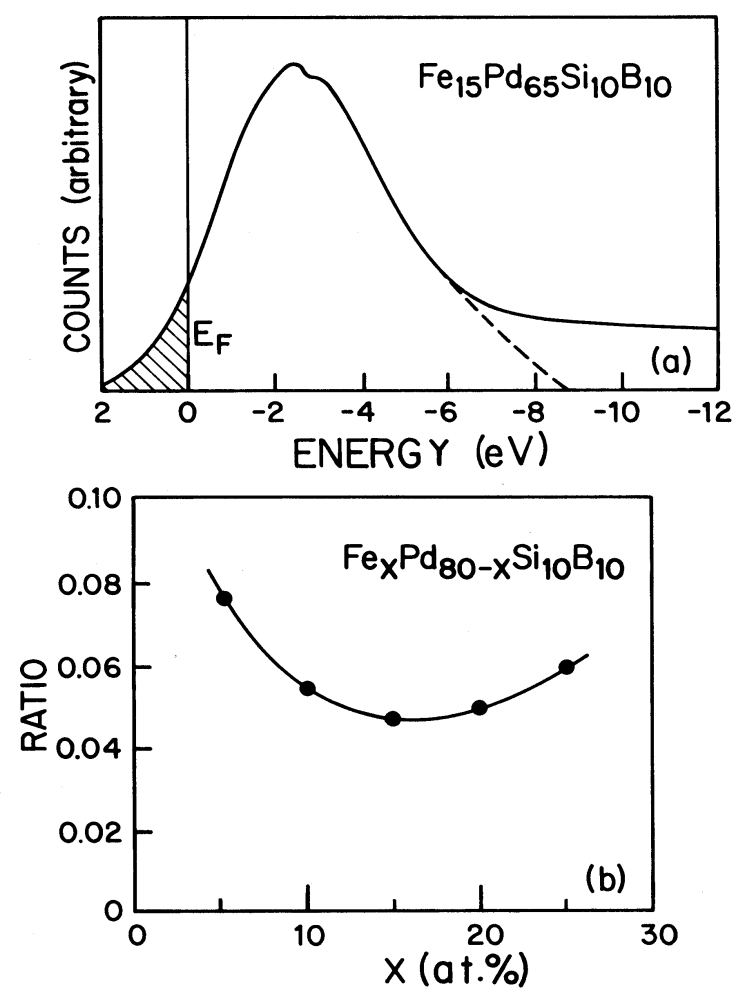

FIG. 5. (a) XPS spectrum of glassy $\mathrm{Fe}_{15} \mathrm{Pd}_{65} \mathrm{Si}_{10} \mathrm{~B}_{10}$. (b) Ratio of crosshatched area in (a) to total valence-band spectrum area, as a function of $\mathrm{Fe}$ concentration (see text).

sitions, paramagnetic-ferromagnetic and ferromagneticspin-glass, exist as the temperature is lowered. The magnetic phase diagram, composition dependence of the saturation magnetization, photoemission, and Mössbauer results all suggest a change in the chemical short-range order or a phase separation for $\mathrm{Fe}$ concentrations above about 13 at. \%. The sharpness of the ferromagnetic transitions for the larger Fe concentrations $(x \geqslant 20)$ is considerably reduced from that of the smaller $\mathrm{Fe}$ concentrations. This also is consistent with the idea that the $x \geqslant 20$ samples have a less random structure than the $x \leqslant 13$ samples. Since many of the metallic glasses studied also contain three or four constituents, microscopic studies of the atomic arrangements in the present and other such glasses will be important in obtaining a detailed understanding of their physical properties.

\section{ACKNOWLEDGMENTS}

We would like to thank Mr. Jim Nabity for preparing the samples and Professor Chen and Ms. Xie for assistance with the susceptibility and XPS measurements, respectively. We are indebted for financial support to the National Science Foundation, under Grant No. DMR-8110520, the University of Nebraska, and the Institute of Physics of the Chinese Academy of Sciences. 
"Present address: The Institute of Physics, Chinese Academy of Sciences, Beijing, China.

${ }^{1}$ R. Hasegawa, J. Appl. Phys. 44, 4096 (1970).

${ }^{2}$ S. R. Nagel, G. B. Fisher, J. Tauc, and B. G. Bagley, Phys. Rev. B 13, 3284 (1970).

${ }^{3}$ U. Mizutani, K. T. Hartwig, T. B. Massalski, and R. W. Hopper, Phys. Rev. Lett. 41, 661 (1978).

${ }^{4}$ P. Oelhafen, M. Laird, H.-J. Güntherodt, K. Berresheim, and H. D. Polaschegg, Solid State Commun. 30, 641 (1979).

${ }^{5}$ C. L. Chien, Phys. Lett. 48A, 394 (1978).

${ }^{6}$ A. Zentko, P. Duhaj, L. Potocky, T. Tima, and J. Bonsky, Phys. Status Solidi (a) 31, K41 (1975).
${ }^{7}$ G. Dublon, C. H. Lin, and J. Bevk, J. Appl. Phys. 50, 7650 (1979).

${ }^{8}$ G. Dublon, Phys. Status Solidi (a) 60, 287 (1980).

${ }^{9} \mathrm{G}$. Dublon and Y. Yeshurun, Phys. Rev. B 25, 4899 (1982).

${ }^{10}$ J. A. Gerber, W. L. Burmester, and D. J. Sellmyer, Rev. Sci. Instrum. 53, 691 (1982).

${ }^{11}$ D. Sherrington and S. Kirkpatrick, Phys. Rev. Lett. 35, 1792 (1975); Phys. Rev. B 17, 4394 (1978).

${ }^{12}$ See, for example, K. V. Rao, Phys. Scr. 25, 742 (1982), and references therein.

${ }^{13}$ T. E. Sharon and C. C. Tsuei, Solid State Commun. 9, 1923 (1971).

14J. G. Zhao, D. J. Sellmyer, and M. Englehardt (unpublished). 